\title{
INTEGRAL REPRESENTATIONS FOR RIESZ SYSTEMS IN THE UNIT BALL AND SOME APPLICATIONS
}

\author{
ASHOT DJRBASHIAN
}

(Communicated by Paul S. Muhly)

\begin{abstract}
We introduce $A_{\alpha}^{p}$ spaces of systems of harmonic functions satisfying Cauchy-Riemann equations in $\mathbf{R}^{\mathbf{n}}$ and find integral representations. Using these representations and estimates for the integral kernel we prove boundedness of the representation operator in $L^{p}$ and Lipschitz classes.
\end{abstract}

\section{INTRODUCTION}

We consider systems of functions $F=\left(f_{1}, \ldots, f_{n}\right)$ satisfying $n$-dimensional Cauchy-Riemann equations in some domain $G \subset \mathbf{R}^{\mathbf{n}}$ :

$$
\sum_{j=1}^{n} \frac{\partial f_{j}}{\partial x_{k}}=0, \quad \frac{\partial f_{j}}{\partial x_{k}}=\frac{\partial f_{k}}{\partial x_{j}}, \quad 1 \leq j, k \leq n .
$$

It is well known (cf. [11]) that equations (1) imply existence (in any connected subdomain of $G$ ) of a harmonic function $H$ such that $F=\nabla H$. In particular, all the components of $F$ are harmonic. We call such vector fields $F$ Riesz systems.

The theory of Hardy spaces $H^{p}$ of Riesz systems in half-spaces is also well known (see [10,11]). This theory is actually based on the notion of Poisson kernel and its conjugate kernels. They play the same role as, in the classical $H^{p}$ theory, the Cauchy kernel and its real and imaginary parts.

However, extension of this theory to other $n$-dimensional domains, including the simplest case of the unit ball, proved to be a very difficult task. It seems that this difficulty is hidden in the problem of finding the conjugates of the Poisson kernel (which itself is classical), while the same thing in the half-space is an easy exercise. This, in its turn, is the consequence of the fact that equations (1) are not rotation invariant. From here it is clear that it is impossible to construct an $H^{p}$ theory in the ball completely analogous to the classical $H^{p}$ theory in the unit disk.

There are two approaches avoiding this difficulty belonging to Korányi and Vági [7] and Ricci and Weiss [9]. We follow mainly the ideas of [7] and construct the theory of more general classes $A_{\alpha}^{p}$ of Riesz systems in the unit ball,

Received by the editors August 2, 1990 and, in revised form, June 5, 1991.

1991 Mathematics Subject Classification. Primary 30D55, 31B05, 42B30, 47B38.

Key words and phrases. Riesz systems, $A_{\alpha}^{p}$ spaces, bounded projections, Lipschitz classes. 
continuing our earlier work [2] where the theory of $A_{\alpha}^{p}$ spaces of Riesz systems in half-spaces was constructed.

This work was announced without proofs in [4] and has been circulating in preprint form since April 1989 (see [5]).

\section{THE INTEGRAL REPRESENTATION FORMULA}

Let $F=\left(f_{1}, \ldots, f_{n}\right)$ be a Riesz system in the unit ball $\mathbf{B}=\left\{x \in \mathbf{R}^{\mathbf{n}}:|x|<\right.$ 1). Following [7] we say that $F \in H^{p}=H^{p}\left(\mathbf{B}, \mathbf{R}^{\mathbf{n}}\right), 0<p \leq \infty$, if

$$
\sup _{0 \leq r<1} \int_{S}\left|F\left(r x^{\prime}\right)\right|^{p} d x^{\prime}<\infty
$$

where $S=\partial \mathbf{B}, x^{\prime} \in S$, and $d x^{\prime}$ is the surface Lebesgue measure on $S$.

The Szegö kernel is the generalization of the classical Cauchy kernel, and we have the following representation formula (see [7]):

Lemma 1. The Szegö kernel $M$ of $H^{2}\left(\mathbf{B}, \mathbf{R}^{\mathbf{n}}\right)$ is given by

$$
M(x, y)=\frac{1}{|S|} \int_{0}^{1} \frac{I-2 r x y^{*}}{|r x-y|^{n}}+n r \frac{\left(y-r|y|^{2} x\right)\left(x-r|x|^{2} y\right)^{*}}{|r x-y|^{n+2}} d r .
$$

Here $I$ is the unit matrix in $\mathbf{R}^{\mathbf{n}}$, the vectors in $\mathbf{R}^{\mathbf{n}}$ are written as columns, and $v^{*}$ is the transpose of $v \in \mathbf{R}^{\mathbf{n}}$.

So we have the following integral representation for $H^{p}$ Riesz systems in the ball: If $F \in H^{p}\left(\mathbf{B}, \mathbf{R}^{\mathbf{n}}\right), 1<p \leq \infty$, then

$$
F(x)=\int_{S} F\left(y^{\prime}\right) M\left(x, y^{\prime}\right) d y^{\prime}
$$

where $F\left(y^{\prime}\right)$ is the boundary value of $F$, which existence is guaranteed by the condition $F \in H^{p}$ (see [7]).

Our aim is to extend this formula to Riesz systems of more general classes, and in order to do it, we begin with some results on $A_{\alpha}^{p}$ spaces from [1] (see also [3]).

A harmonic function $f$ in $\mathbf{B}$ belongs to the class $A_{\alpha}^{p}=A_{\alpha}^{p}(\mathbf{B}), 0<p<\infty$, $-1<\alpha<\infty$, if

$$
\|f\|_{p, \alpha}^{p}=\int_{\mathbf{B}}|f(x)|^{p}(1-|x|)^{\alpha} d x<\infty
$$

where $d x$ is the solid Lebesgue measure in $\mathbf{R}^{\mathbf{n}}$.

Analogously, a vector function $F=\left(f_{1}, \ldots, f_{n}\right)$ is in $A_{\alpha}^{p}\left(\mathbf{B}, \mathbf{R}^{\mathbf{n}}\right)$ if $F$ is a Riesz system and $F(x)=\left(\sum\left|f_{j}(x)\right|^{2}\right)^{1 / 2}$ satisfies $(5)$.

Now we present two results from [1] (see also [3, Chapter 7]). These results are slightly modified in view of their further use. The proofs of the modified versions, however, are identical with the original ones.

Theorem 1. If the (scalar) function $f \in A_{\alpha}^{p}, 1 \leq p<\infty, \alpha>-1$, then the following integral representation holds:

$$
f(x)=\int_{\mathbf{B}} f(y) Q_{\alpha}(x, y)(1-|y|)^{\alpha} d y .
$$

Here

$$
Q_{\alpha}(x, y)=\sum_{k \geq 0} \frac{\Gamma(2 k+n+1+\alpha)}{\Gamma(1+\alpha) \Gamma(2 k+n)}(|x||y|)^{k} Z_{x^{\prime}}^{(k)}\left(y^{\prime}\right)
$$


where $Z^{(k)}$ is the so-called zonal spherical harmonic of order $k \geq 0, x^{\prime}=x /|x|$, $y^{\prime}=y /|y| \in S$.

The precise form of the zonal harmonic is not essential for the time being. A little later we will come back to it.

Lemma 2. If $\alpha=m \geq 0$ is an integer then

$$
\left|Q_{m}(x, y)\right| \leq C|x| y\left|-y^{\prime}\right|^{-n-m} .
$$

Now recall some classical facts.

Let $H$ be a Hilbert space of functions in some domain $G$. Representing kernel of $H$ is defined to be a function $K(x, y)$ defined in $G \times G$ such that $f(x)=(K(x, y), f(y))$ for any $f \in H$ and $(.,$.$) is the inner product in H$.

Lemma $3[7,12]$. Let $H$ be a separable Hilbert space and suppose $\left\{\psi_{k}\right\}_{k \geq 1}$ is an orthonormal basis in $H$. The representing kernel for $H$ exists if and only if for any $x_{0} \in G$ there exists a constant $C=C_{x_{0}}$ such that $\left|f\left(x_{0}\right)\right| \leq C\|f\|_{H}$. In that case this representing kernel is given by the series

$$
K(x, y)=\sum_{k \geq 1} \psi_{k}^{*}(y) \psi_{k}(x),
$$

where $\psi_{k}^{*}$ is the conjugate to the element $\psi_{k}$.

Turning back to spherical functions, we recall that restriction of any homogeneous polynomial of degree $k \geq 0$ in B to the unit sphere $S$ is called a spherical harmonic of degree $k$. For any $k \geq 0$ there exist $d_{k}=$ $(2 k+n-2)(n+k-3) ! /(n-2) ! k$ ! linearly independent spherical harmonics of degree $k$. We denote them by $Y_{j}^{(k)}, 1 \leq j \leq d_{k}$.

The zonal harmonic $Z^{(k)}$ (see Theorem 1) has the expansion

$$
Z_{x^{\prime}}^{(k)}\left(y^{\prime}\right)=\sum_{j=1}^{d_{k}} Y_{j}^{(k)}\left(x^{\prime}\right) Y_{j}^{(k)}\left(y^{\prime}\right) .
$$

(For these and other results on harmonics see, e.g., [11].)

The next result is from [7, p. 186].

Lemma 4. Let $\left\{Y_{j}^{(k)}\right\}, k \geq 0,1 \leq j \leq d_{k}$, be the complete orthonormal system of spherical harmonics in $L^{2}(S)$. Then the system

$$
\left\{\boldsymbol{\Phi}_{k j}(x)\right\}=\left\{(k(2 k+n-2))^{-1 / 2} \nabla\left(|x|^{k} Y_{j}^{(k)}\left(x^{\prime}\right)\right)\right\}, \quad k \geq 1,1 \leq j \leq d_{k},
$$

is complete and orthonormal in $H^{2}\left(\mathbf{B}, \mathbf{R}^{\mathbf{n}}\right)$.

Now we can construct an orthonormal basis for $A_{\alpha}^{2}\left(\mathbf{B}, \mathbf{R}^{\mathbf{n}}\right)$.

\section{Lemma 5.}

$$
\begin{aligned}
&\left\{\varphi_{k j}(x)\right\}=\left\{\left(\frac{\Gamma(2 k+n-1+\alpha)}{\Gamma(1+\alpha) \Gamma(2 k+n-1) k}\right)^{1 / 2} \nabla\left(|x|^{k} Y_{j}^{(k)}\left(x^{\prime}\right)\right)\right\} \\
& k \geq 1,1 \leq j \leq d_{k},
\end{aligned}
$$

is an orthonormal basis in $A_{\alpha}^{2}\left(\mathbf{B}, \mathbf{R}^{\mathbf{n}}\right)$. 
Proof. From the proof of the previous lemma, we know that if $u(x)=$ $|x|^{k} Y_{j}^{(k)}\left(x^{\prime}\right)$ for some $k \geq 1,1 \leq j \leq d_{k}$, then

$$
\|\nabla u\|_{H^{2}\left(\mathbf{B}, \mathbf{R}^{\mathbf{n}}\right)}^{2}=\int_{S}\left|\nabla u\left(x^{\prime}\right)\right|^{2} d x^{\prime}=k(2 k+n-2) .
$$

Hence, observing that $|\nabla u|$ is homogeneous of order $k-1$, we get

$$
\begin{aligned}
\|\nabla u\|_{A_{\alpha}^{2}\left(\mathbf{B}, \mathbf{R}^{\mathbf{R}}\right)}^{2} & =\int_{\mathbf{B}}\left|\nabla u\left(r x^{\prime}\right)\right|^{2}(1-r)^{\alpha} r^{n-1} d r d x^{\prime} \\
& =\int_{0}^{1}(1-r)^{\alpha} r^{2 k-2+n-1} d r \int_{S}\left|\nabla u\left(x^{\prime}\right)\right|^{2} d x^{\prime} \\
& =\frac{\Gamma(1+\alpha) \Gamma(2 k+n-2)}{\Gamma(2 k+n-1+\alpha)} \cdot k(2 k+n-2) \\
& =\frac{\Gamma(1+\alpha) \Gamma(2 k+n-1) k}{\Gamma(2 k+n-1+\alpha)} .
\end{aligned}
$$

Completeness of the system $\left\{\varphi_{k j}\right\}$ in $A_{\alpha}^{2}\left(\mathbf{B}, \mathbf{R}^{\mathbf{n}}\right)$ follows from the corresponding place in the proof of the previous lemma.

Using Lemmas 3 and 5 we immediately get

Theorem 2. The representing kernel of the space $A_{\alpha}^{2}\left(\mathbf{B}, \mathbf{R}^{\mathbf{n}}\right)$ is a matrix-function given by the series

$$
K^{(\alpha)}(x, y)=\sum_{k, j} \varphi_{k j}^{*}(y) \varphi_{k j}(x) .
$$

In other words, if $F \in A_{\alpha}^{2}\left(\mathbf{B}, \mathbf{R}^{\mathbf{n}}\right), \alpha>-1$, then

$$
F(x)=\int_{\mathbf{B}} F(y) K^{(\alpha)}(x, y)(1-|y|)^{\alpha} d y .
$$

Using the obvious fact that $A_{\alpha}^{2} \cap A_{\alpha}^{p}$ is dense in $A_{\alpha}^{p}, 1 \leq p<\infty$, we get that any function $F \in A_{\alpha}^{p}\left(\mathbf{B}, \mathbf{R}^{\mathbf{n}}\right)$ also has the representation (9). Moreover, since for $0<p<1, A_{\alpha}^{p}\left(\mathbf{B}, \mathbf{R}^{\mathbf{n}}\right) \subset A_{\beta}^{1}\left(\mathbf{B}, \mathbf{R}^{\mathbf{n}}\right)$, for any $\beta>(n+\alpha) / p-1$, we get that a representation analogous to (9) is true even for $A_{\alpha}^{p}$ with $0<p<1$. The only difference is that in (9) one must change $\alpha$ by $\beta$ with $\beta>(n+\alpha) / p-1$.

Next, if $1<p<\infty, \alpha>-1$, then by Hölder's inequality $A_{\alpha}^{p} \subset A_{\beta}^{1}$ for $\beta>(1+\alpha) / p-1$. Combining all these we have

Theorem 3. Let $F \in A_{\alpha}^{p}\left(\mathbf{B}, \mathbf{R}^{\mathbf{n}}\right), 0<p<\infty, \alpha>-1$. Suppose also that $\beta>(n+\alpha) / p-1$ if $0<p<1 ; \beta \geq \alpha$ if $p=1 ; \beta>(1+\alpha) / p-1$ if $1<p<\infty$. Then we have the representation

$$
F(x)=\int_{\mathbf{B}} F(y) K^{(\beta)}(x, y)(1-|y|)^{\beta} d y .
$$

In order to prove further results on $A_{\alpha}^{p}$ functions we need effective estimates for the kernel $K^{(\alpha)}$. However, if we try to do it for arbitrary parameter $\alpha>-1$, it will be very difficult technically and will not give satisfactory results. So, we will restrict ourselves to the case of integer $\alpha$ 's. In view of the previous theorem we will not lose generality since for any $\alpha>-1$ we have infinitely many representations depending on discrete parameter $m$. 
Lemma 6. Let $m \geq 0$ be an integer. Then for $1 \leq j, k \leq n$, the elements $K_{j k}^{(m)}(x, y)$ of the kernel $K^{(m)}(x, y)$ satisfy

$$
\left|K_{j k}^{(m)}(x, y)\right| \leq C\left|r \rho x^{\prime}-y^{\prime}\right|^{-n-m},
$$

where $x, y \in \mathbf{B}, x=r x^{\prime}, y=\rho y^{\prime}$.

Proof. By Lemma 5 we have

$$
\begin{aligned}
K^{(\alpha)}(x, y) & =\sum_{k, j} \frac{\Gamma(2 k+n+\alpha-1)}{\Gamma(1+\alpha) \Gamma(2 k+n-1) k}\left(\nabla \rho^{k} Y_{j}^{(k)}\left(y^{\prime}\right)\right)^{*}\left(\nabla r^{k} Y_{j}^{(k)}\left(x^{\prime}\right)\right) \\
& =\nabla_{y} \nabla_{x} \sum_{k \geq 1} \frac{\Gamma(2 k+n+\alpha-1)}{\Gamma(2 k+n-1) \Gamma(1+\alpha) k}(r \rho)^{k} \sum_{j=1}^{d_{k}} Y_{j}^{(k)}\left(y^{\prime}\right) Y_{j}^{(k)}\left(x^{\prime}\right) \\
& =\nabla_{y} \nabla_{x} \sum_{k \geq 1} \frac{\Gamma(2 k+n+\alpha-1)}{\Gamma(2 k+n-1) \Gamma(1+\alpha) k}(r \rho)^{k} Z_{x^{\prime}}^{(k)}\left(y^{\prime}\right) .
\end{aligned}
$$

Denoting this last sum by $M^{(\alpha)}(x, y)$, we obviously have

$$
K_{l i}^{(\alpha)}(x, y)=\frac{\partial}{\partial y_{l}} \frac{\partial}{\partial x_{i}} M^{(\alpha)}(x, y),
$$

and the problem is reduced to the computation of the function $M^{(\alpha)}$ in the case of integer $\alpha=m \geq 0$.

Consider the case $m \geq 1$ first. We have

$$
\begin{aligned}
M^{(m)}(x, y) & =\sum_{k \geq 1} \frac{(2 k+n+m-2) !}{(2 k+n-2) ! m ! k}(r \rho)^{k} Z_{x^{\prime}}^{(k)}\left(y^{\prime}\right) \\
& =\sum_{k \geq 1} \frac{(2 k+n+m-2) !(k-1) !}{(2 k+n-2) !(k+m-1) ! m !} \frac{d^{m-1}}{d(r \rho)^{m-1}}(r \rho)^{k+m-1} Z_{x^{\prime}}^{(k)}\left(y^{\prime}\right) .
\end{aligned}
$$

By Stirling's formula, $(2 k+n+m-2) !(k-1) ! /(2 k+n-2) !(k+m-1) ! \rightarrow 2^{m}$ as $k \rightarrow \infty$ and hence, we can continue, denoting also $r \rho=t$,

$$
\begin{aligned}
& M^{(m)}(x, y)= \sum_{k \geq 1} \frac{2^{m}}{m !} \frac{d^{m-1}}{d t^{m-1}} t^{k+m-1} Z_{x^{\prime}}^{(k)}\left(y^{\prime}\right) \\
&+\frac{1}{m !} \sum_{k \geq 1}\left(\frac{(2 k+n+m-2) !(k-1) !}{(2 k+n-2) !(k+m-1) !}-2^{m}\right) \\
& \times \frac{d^{m-1}}{d t^{m-1}} t^{k+m-1} Z_{x^{\prime}}^{(k)}\left(y^{\prime}\right) .
\end{aligned}
$$

Let us treat the second term first. We have

$$
(2 k+n+m-2) !(k-1) ! /(2 k+n-2) !(k+m-1) !-2^{m} \asymp k^{-1}
$$

and

$$
\sum_{k \geq 1} k^{-1} \frac{d^{m-1}}{d t^{m-1}} t^{k+m-1} z_{x^{\prime}}^{(k)}\left(y^{\prime}\right) \asymp \frac{d^{m-2}}{d t^{m-2}} \sum_{k \geq 1} t^{k+m-1} Z_{x^{\prime}}^{(k)}\left(y^{\prime}\right) .
$$


Hence, the second term on the right side of (12) has lower order of growth than the first one, and so we can write

$$
M^{(m)}(x, y)=\frac{2^{m}}{m !} \frac{d^{m-1}}{d t^{m-1}}\left(t^{m-1} \sum_{k \geq 1} t^{k} Z_{x^{\prime}}^{(k)}\left(y^{\prime}\right)\right)+\text { Lower Order Terms. }
$$

Recall the spherical harmonics expansion of the Poisson kernel in B (see [11, Chapter 4])

$$
P\left(t x^{\prime}, y^{\prime}\right)=\sum_{k \geq 0} t^{k} Z_{x^{\prime}}^{(k)}\left(y^{\prime}\right)
$$

Consequently,

$$
M^{(m)}(x, y)=\frac{2^{m}}{m !} \frac{d^{m-1}}{d t^{m-1}}\left(t^{m-1} P\left(t x^{\prime}, y^{\prime}\right)-t^{m-1}\right)+\text { L.O.T. }
$$

Finally,

$$
K_{j k}^{(m)}(x, y)=\frac{2^{m}}{m !} \frac{\partial}{\partial y_{j}} \frac{\partial}{\partial x_{k}} \frac{d^{m-1}}{d(r)^{m-1}}\left((r \rho)^{m-1} P\left(r x^{\prime}, y^{\prime}\right)-(r \rho)^{m-1}\right)+\text { L.O.T. }
$$

Now, using Lemma 7.1 of [3] for differentiation of the Poisson kernel, we get the proof of our assertion.

To conclude the proof of the lemma it remains to consider the case $m=0$ only. In this case

$$
M^{(0)}(x, y)=\sum_{k \geq 1} k^{-1}(r \rho)^{k} Z_{x^{\prime}}^{(k)}\left(y^{\prime}\right)
$$

and this last sum is equivalent to the integral of the Poisson kernel. Hence, taking partial derivatives by $x_{k}$ and $y_{j}$, we again arrive to the conclusion of the lemma.

Remark. Proceeding in the same way, we find that the partial derivatives of the kernels $K_{j k}^{(m)}, 1 \leq j, k \leq n$, satisfy the estimates

$$
\left|\frac{\partial}{\partial x_{i}} K_{j k}^{(m)}(x, y)\right| \leq C\left|r \rho x^{\prime}-y^{\prime}\right|^{-n-m-1}, \quad 1 \leq i \leq n,
$$

and the same estimates are true for derivatives by $y_{i}$.

\section{BOUNDEDNESS OF THE PROJECTION OPERATOR AND PRESERVATION OF LIPSCHITZ CLASSES}

As it is seen by Lemma 6 , the elements of the matrix kernel $K^{(m)}$ satisfy estimates completely analogous to that of the kernel $Q_{m}$ of scalar functions (see Lemma 2). Hence we can hope that many results for scalar functions could be extended to $A_{\alpha}^{p}$ classes of Riesz systems. In particular, we will prove the projection theorem; we need one more lemma.

Lemma 7. For fixed $1 \leq k \leq n$ the vector $\left(K_{1 k}^{(\alpha)}, \ldots, K_{n k}^{(\alpha)}\right)$ is a Riesz system in the variable $y$, and for fixed $1 \leq j \leq n$ the vector $\left(K_{j 1}^{(\alpha)}, \ldots, K_{j n}^{(\alpha)}\right)$ is a Riesz system in $x$.

The proof is an easy exercise in the change of order of differentiation and we omit it. 
Denote by $L_{\alpha}^{p}\left(\mathbf{B}, \mathbf{R}^{\mathbf{n}}\right)$ the space of all real measurable vector functions $G(x)=\left(g_{1}(x), \ldots, g_{n}(x)\right)$ such that $|G|=\left(\sum\left|g_{j}\right|^{2}\right)^{1 / 2}$ belongs to the class $L^{p}\left(\mathbf{B},(1-|x|)^{\alpha} d x\right), 0<p \leq \infty, \alpha>-1$.

Note that we do not demand that $G$ be a Riesz system or even that the components $g_{j}$ be harmonic.

Now let $G \in L_{\alpha}^{p}\left(\mathbf{B}, \mathbf{R}^{\mathbf{n}}\right)$ and consider the integral

$$
T_{m} G(x)=\int_{\mathbf{B}} G(y) K^{(m)}(x, y)(1-|y|)^{m} d y .
$$

It is not clear a priori that this integral is well defined for arbitrary vector function from $L_{\alpha}^{p}\left(\mathbf{B}, \mathbf{R}^{\mathbf{n}}\right)$. However, using estimates (11) we see that it is the case if $1 \leq p \leq \infty, \alpha>-1$, and $m$ satisfies certain conditions. Moreover, it is obvious that the vector function $T_{m} G$ is harmonic. As in the case of scalar functions, the following result is true.

Theorem 4. Let $1 \leq p<\infty, \alpha>-1$, and $m>(1+\alpha) / p-1$. Then the operator $T_{m}$ defined by the formula (14) is a bounded projection from $L_{\alpha}^{p}\left(\mathbf{B}, \mathbf{R}^{\mathbf{n}}\right)$ to its subspace $A_{\alpha}^{p}\left(\mathbf{B}, \mathbf{R}^{\mathbf{n}}\right)$.

Proof. Let $G=\left(g_{1}, \ldots, g_{n}\right) \in L_{\alpha}^{p}\left(\mathbf{B}, \mathbf{R}^{\mathbf{n}}\right), 1 \leq p<\infty, \alpha>-1$, and denote $F=T_{m} G=\left(f_{1}, \ldots, f_{n}\right)$. Then

$$
f_{k}(x)=\int_{\mathbf{B}} \sum_{j=1}^{n} g_{j}(y) K_{j k}^{(m)}(x, y)(1-|y|)^{m} d y, \quad 1 \leq k \leq n .
$$

It is clear that every $f_{k}$ is harmonic. Moreover, by Lemma 7, $F$ is a Riesz system. Now the proof is reduced to the scalar case since any integral

$$
\int_{\mathbf{B}} g_{j}(y) K_{j k}^{(m)}(x, y)(1-|y|)^{m} d y
$$

is an $A_{\alpha}^{p}$ scalar function by Lemma 6 and the corresponding result for scalar functions (see $[1,3])$.

Next we will consider the problem of preservation of Lipschitz classes under the action of the integral operator (14).

Definition. A function $f(x)$ defined in $\mathbf{B}$ is in the Lipschitz class $\operatorname{Lip} \beta, 0<$ $\beta<1$, if $f$ is continuous in $\overline{\mathbf{B}}$ and for any $x, y \in \overline{\mathbf{B}},|f(x)-f(y)| \leq C|x-y|^{\beta}$.

A vector-valued function $F=\left(f_{1}, \ldots, f_{n}\right)$ belongs to the class $\operatorname{Lip} \beta\left(\mathbf{B}, \mathbf{R}^{\mathbf{n}}\right)$ if any component $f_{j}, 1 \leq j \leq n$, is in $\operatorname{Lip} \beta(\mathbf{B})$.

The following is an analogue of the well-known Hardy-Littlewood theorem (see, e.g., [6] for the classical result).

Lemma 8. Let $f$ be harmonic in $\mathbf{B}$. Then $f$ is continuous in $\overline{\mathbf{B}}$ and belongs to $\operatorname{Lip} \beta, 0<\beta<1$ in $\overline{\mathbf{B}}$ if and only if

$$
|\nabla f(x)| \leq C(1-|x|)^{\beta-1} .
$$

The proof is a repetition of the classical one with obvious changes.

It follows from this lemma that, equivalently, a harmonic vector function $F \in \operatorname{Lip} \beta\left(\mathbf{B}, \mathbf{R}^{\mathbf{n}}\right)$ if and only if $\left|\nabla f_{j}(x)\right| \leq C(1-|x|)^{\beta-1}, 1 \leq j \leq n$. 
Theorem 5. The operator $T_{m}$ defined by (14) for an integer $m \geq 0$ preserves Lipschitz classes. More precisely, if $G$ is a Lipschitz vector function with $0<$ $\beta<1$ (not necessarily harmonic) then

$$
T_{m} G(x)=\int_{\mathbf{B}} G(y) K^{(m)}(x, y)(1-|y|)^{m} d y
$$

is a Riesz system of the class $\operatorname{Lip} \beta\left(\mathbf{B}, \mathbf{R}^{\mathbf{n}}\right), 0<\beta<1$. Moreover, there exists a constant $C>0$ depending only on $n, m$, and $\beta$ so that

$$
\left\|T_{m} G\right\|_{\operatorname{Lip} \beta} \leq C\|G\|_{\operatorname{Lip} \beta} .
$$

Proof. By Lemma 8 and the remark just before the theorem it is sufficient to prove that

$$
\left|\frac{\partial}{\partial x_{i}} f_{k}(x)\right| \leq C(1-|x|)^{\beta-1}, \quad 1 \leq i, k \leq n,
$$

where $F=T_{m} G=\left(f_{1}, \ldots, f_{n}\right)$.

For arbitrary $z \in \mathbf{B}$ with $|z-x| \leq 1-|x|, z \neq x$, we have

$$
\begin{aligned}
f_{k}(x) & =\int_{\mathbf{B}} \sum_{j=1}^{n} K_{j k}^{(m)}(x, y) g_{j}(y)(1-|y|)^{m} d y \\
& =\int_{\mathbf{B}} \sum_{j=1}^{n} K_{j k}^{(m)}(x, y)\left(g_{j}(y)-g_{j}(z)\right)(1-|y|)^{m} d y+g_{k}(z),
\end{aligned}
$$

since any scalar vector is a Riesz system. Hence, using (13),

$$
\begin{aligned}
\left|\frac{\partial f_{k}}{\partial x_{i}}(x)\right| & \leq \int_{\mathbf{B}} \sum_{j=1}^{n}\left|\frac{\partial}{\partial x_{i}} K_{j k}^{(m)}(x, y)\right|\left|g_{j}(y)-g_{j}(z)\right|(1-|y|)^{m} d y \\
& \leq C \int_{\mathbf{B}} \frac{|y-z|^{\beta}}{|x| y\left|-y^{\prime}\right|^{n+m+1}}(1-|y|)^{m} d y \\
& \leq C \int_{\mathbf{B}} \frac{|y-z|^{\beta} d y}{|x| y\left|-y^{\prime}\right|^{n+1}} \\
& \leq C \int_{\mathbf{B}} \frac{|y-x|^{\beta} d y}{|x| y\left|-y^{\prime}\right|^{n+1}}+C \int_{\mathbf{B}} \frac{|z-x|^{\beta} d y}{|x| y\left|-y^{\prime}\right|^{n+1}} \\
& \leq C \int_{\mathbf{B}} \frac{d y}{|x| y\left|-y^{\prime}\right|^{n+1-\beta}}+C(1-|x|)^{\beta} \int_{\mathbf{B}} \frac{d y}{|x| y\left|-y^{\prime}\right|^{n+1}}
\end{aligned}
$$

since $|y-x| \leq C|x| y\left|-y^{\prime}\right|$ and $|z-x| \leq 1-|x|$ by our choice. Finally,

$$
\left|\frac{\partial}{\partial x_{i}} f_{k}(x)\right| \leq C(1-|x|)^{\beta-1}+C(1-|x|)^{\beta} \frac{C}{1-|x|}=C(1-|x|)^{\beta-1},
$$

and the theorem is proved.

Remark. Theorem 5 is new even for scalar functions. For similar results for holomorphic functions in strictly pseudoconvex domains and further references see [8].

\section{ACKNOWLEDGMENTS}

I am grateful to Professors A. Korányi and G. Weiss who in response to my question (Notices Amer. Math. Soc. 33 (1986), 519) sent me their papers [7] and [9], respectively. 


\section{REFERENCES}

1. A. E. Djrbashian, Integral representations and continuous projections in certain spaces of harmonic functions, Math. USSR-Sb. 121 (1983), 259-271. (Russian)

2. —,$A_{\alpha}^{p}$ classes of harmonic functions in half-spaces and an analogue of $M$. Riesz theorem, Izv. Acad. Sci. Armenian SSR Ser. Math. 22 (1987), 386-398. (Russian)

3. A. E. Djrbashian and F. A. Shamoian, Topics in the theory of $A_{\alpha}^{p}$ spaces, Teubner Texte zur Math., vol. 105, Teubner-Verlag, Leipzig, 1988.

4. A. E. Djrbashian, Integral representations for classes of harmonic vector functions in the unit ball, Dokl. Acad. Sci. Armenian SSR 88 (1989), 112-116. (Russian)

5. __ Integral representations for Riesz systems in half-spaces and balls, preprint 89-01, Inst. Math. Acad. Sci. Armenian SSR, Yerevan 1989.

6. P. L. Duren, Theory of $H^{p}$ spaces, Academic Press, New York and London, 1970.

7. A. Korányi and S. Vági, Cauchy-Szegö integrals for systems of harmonic functions, Ann. Scuola Norm. Sup. Pisa Cl. Sci. (4) 26 (1972), 181-196.

8. E. Ligocka, The Hölder continuity of the Bergman projection and proper holomorphic mappings, Studia Math. 80 (1984), 89-107.

9. F. Ricci and G. Weiss, $A$ characterization of $H^{1}\left(\sum_{n-1}\right)$, Proc. Sympos. Pure Math., vol. 35, Amer. Math. Soc., Providence, RI, 1975, pp. 289-294.

10. E. M. Stein, Singular integrals and differentiability properties of functions, Princeton Univ. Press, Princeton, NJ, 1970.

11. E. M. Stein and G. Weiss, Introduction to Fourier analysis on euclidean space, Princeton Univ. Press, Princeton, NJ, 1971.

12. K. Yosida, Functional analysis, Springer-Verlag, Berlin, 1965.

817 1/2 North Heliotrope Drive, Los ANgeles, California 90029 